\title{
Metabolic Syndrome and its Association with Healthy Eating Index-2005 in Adolescents: Tehran Lipid and Glucose Study
}

\author{
Sahar Mohseni-Takalloo ${ }^{1,2}$, Parvin Mirmiran ${ }^{1,3, *}$, Firoozeh Hosseini-Esfahani ${ }^{1}$, Yadollah Mehrabi ${ }^{4}$, Fereidoun \\ Azizi $^{5}$ \\ ${ }^{1}$ Nutrition and Endocrine Research Center, Research Institute for Endocrine Sciences, Shahid Beheshti University of Medical Sciences, \\ Tehran, Iran \\ ${ }^{2}$ Faculty of School of Medicine, Bam University of Medical Sciences, Bam, Iran \\ ${ }^{3}$ Faculty of Nutrition Sciences and Food Technology, National Nutrition and Food Technology Research Institute, Shahid Beheshti \\ University of Medical Sciences, Tehran, Iran \\ ${ }^{4}$ Department of Epidemiology, Faculty of Public Health, Shahid Beheshti University of Medical Sciences, Tehran, Iran \\ ${ }^{5}$ Endocrine Research Center, Research Institute for Endocrine Sciences, Shahid Beheshti University of Medical Sciences, Tehran, Iran \\ *Corresponding author: Mirmiran@endocrine.ac.ir
}

Received March 13, 2014; Revised April 26, 2014; Accepted May 03, 2014

\begin{abstract}
One of the major epidemiologic public health problems is worldwide metabolic syndrome (MetS). Since the relationship between diet quality and MetS among adolescents in developing countries has not been well understood, the present study aimed to evaluate the association between diet quality, as measured by Healthy Eating Index (HEI-2005) and the prevalence of MetS and its risk factors in a group of Tehranian adolescents. This crosssectional study was conducted on 706 adolescents, aged 10-19 years, within the framework of Tehran Lipid and Glucose Study in 2008-2011. Usual dietary intakes were assessed by valid and reliable semi-quantitative food frequency questionnaire and diet quality score was obtained based on HEI-2005. MetS was determined using the modified de Ferranti definition. There were 706 adolescents, aged $14.8 \pm 2.7$ years. The overall prevalence of MetS in adolescents was $22.4 \%$. Large waist circumference was the most common component of MetS in both girls and boys. After adjusting for age, sex, energy intake and physical activity, participants who were in the highest quartile of HEI-2005 compared with lowest quartile had lower prevalence of hypertriglyceridemia (OR: 0.62; CI 95\%: 0.400.96 and $\mathrm{P}$ for trend= 0.02), and low serum HDL-C (OR: 0.62; CI 95\%: 0.41-0.97 and P for trend= 0.02). No significant difference was observed in odds of MetS, large waist circumference, hyperglycemia and hypertension. Adolescents with dietary pattern more consistent with the HEI-2005, had lower prevalence of hypertriglyceridemia and low serum HDL-C.
\end{abstract}

Keywords: diet quality, metabolic syndrome, adolescent, dietary guidelines, healthy eating index-2005

Cite This Article: Sahar Mohseni-Takalloo, Parvin Mirmiran, Firoozeh Hosseini-Esfahani, Yadollah Mehrabi, and Fereidoun Azizi, "Metabolic Syndrome and its Association with Healthy Eating Index-2005 in Adolescents: Tehran Lipid and Glucose Study.” Journal of Food and Nutrition Research, vol. 2, no. 4 (2014): 155-161. doi: 10.12691/jfnr-2-4-4.

\section{Introduction}

Metabolic syndrome (MetS) is defined as a cluster of four major metabolic abnormalities, including central obesity, hypertension, dyslipidemia and disturbed glucose homeostasis [1]. Obesity, MetS and type 2 diabetes have emerged as worldwide epidemiologic public health problems [2]. It has been shown that the prevalence of MetS in adolescents increases with progressive increase in the prevalence of overweight and obesity [3]. Like other developing countries, Iran is also experiencing global epidemic obesity and its consequences [4]. The prevalence of MetS among Iranian children and adolescent was 14\% ${ }^{[5]}$, and among Tehranian adolescents was $6.7 \%$, between
1999 and 200 [6] and 9.5\% between 2005 and 2008 [7]. However, it is difficult to compare findings of different researches due to the lack of consensus in metabolic syndrome definition in adolescent [1]. Data reports that raise in MetS in adolescent leads to an increase in coronary heart complications and type 2 diabetes in adulthood [8].

Of the factors affecting the prevalence of metabolic syndrome, special attention is paid to diet quality as a controllable environmental factor [9]. Two methods are commonly used in the study to evaluate the dietary patterns. First, evaluate dietary patterns based on statistical modeling of available dietary data such as factor analysis and cluster analysis. The second approach assesses a predetermined dietary pattern that is based on previous knowledge of a healthy diet, like diet quality 
indices [10]. The American Heart Association recommended adherence to the dietary guidelines for Americans (DGA) to decrease the risk of Mets [11]. DGA-2005, an updated version of DGA, was published with new concepts of diet in 2005 [12],emphasizing on important aspects of diet quality such as whole grain, various types of vegetables and specific types of fat, and introducing the new concept of "discretionary calories". The Healthy Eating Index-2005 (HEI-2005), developed by revision of the original HEI, measures diet quality based on the key dietary recommendations of the DGA-2005 [13]. One study has shown a reverse relationship between MetS prevalence and HEI scores in US adolescents [14]; it has also been reported that higher HEI score was associated with decrease in number of MetS risk factors presented in children and adolescents in Crete [1].

To the best of our knowledge, no study has examined the relationship between MetS prevalence and the updated version of DGA in Iranian adolescents. The aim of the present study was to evaluate the association of adherence to HEI-2005 and prevalence of MetS and its risk factors in Tehranian adolescents.

\section{Methods and Materials}

\subsection{Study Population}

The Tehran Lipid and Glucose Study (TLGS) is an ongoing prospective population based investigation aimed at detecting and preventing non-communicable diseases and their risk factors in residents of district no. 13 of Tehran, the capital city of Iran $[15,16]$.
During the fourth phase of TLGS (2008-2011), a total of 1454 adolescents, aged 10 to 19 years completed the examination, of which 848 were randomly selected for completing the dietary assessment. Subject were excluded from the present study if they (a) lacked any completed anthropometric, biochemical or physical activity data $(n=6)$, (b) were taking medications classified as blood glucose, lipid or blood pressure regulators $(n=23)$, (c) had special diets $(n=16)$ or $(d)$ reported energy intake $\leq 800$ $\mathrm{kcal}$ or $\geq 5000 \mathrm{kcal}$ for boys and $\leq 600 \mathrm{kcal}$ or $\geq 4500 \mathrm{kcal}$ for girls $(n=97)$ (estimated dietary intake most likely unreliable) [17]. Finally, the data for 706 adolescents (385 girls and 321 boys) were analyzed.

This study protocol was approved by the research council of the Research Institute for Endocrine Science, written informed consent was obtained from all those aged over 18 years and from the parents of those aged $<18$ years.

\subsection{Dietary Intake Assessment}

Dietary data were collected using a validated 168-item semi-quantitative Food Frequency Questionnaire (FFQ) $[18,19,20]$. Trained dietitians asked participants to report their frequency of consumption of each food item, during a year on a daily, weekly or monthly basis. Since the Iranian Food Composition Table (FCT) is incomplete with limited data on nutrient content of raw food and beverages [21], we used the FCT of the US Department of Agriculture (USDA) [22] in our study. Moreover, for mixed food items (e.g. pizza), nutrients were calculated according to usual restaurant recipes.

Table 1. Healthy Eating Index $\mathbf{- 2 0 0 5}$ components and standards for scoring ${ }^{\text {a }}$

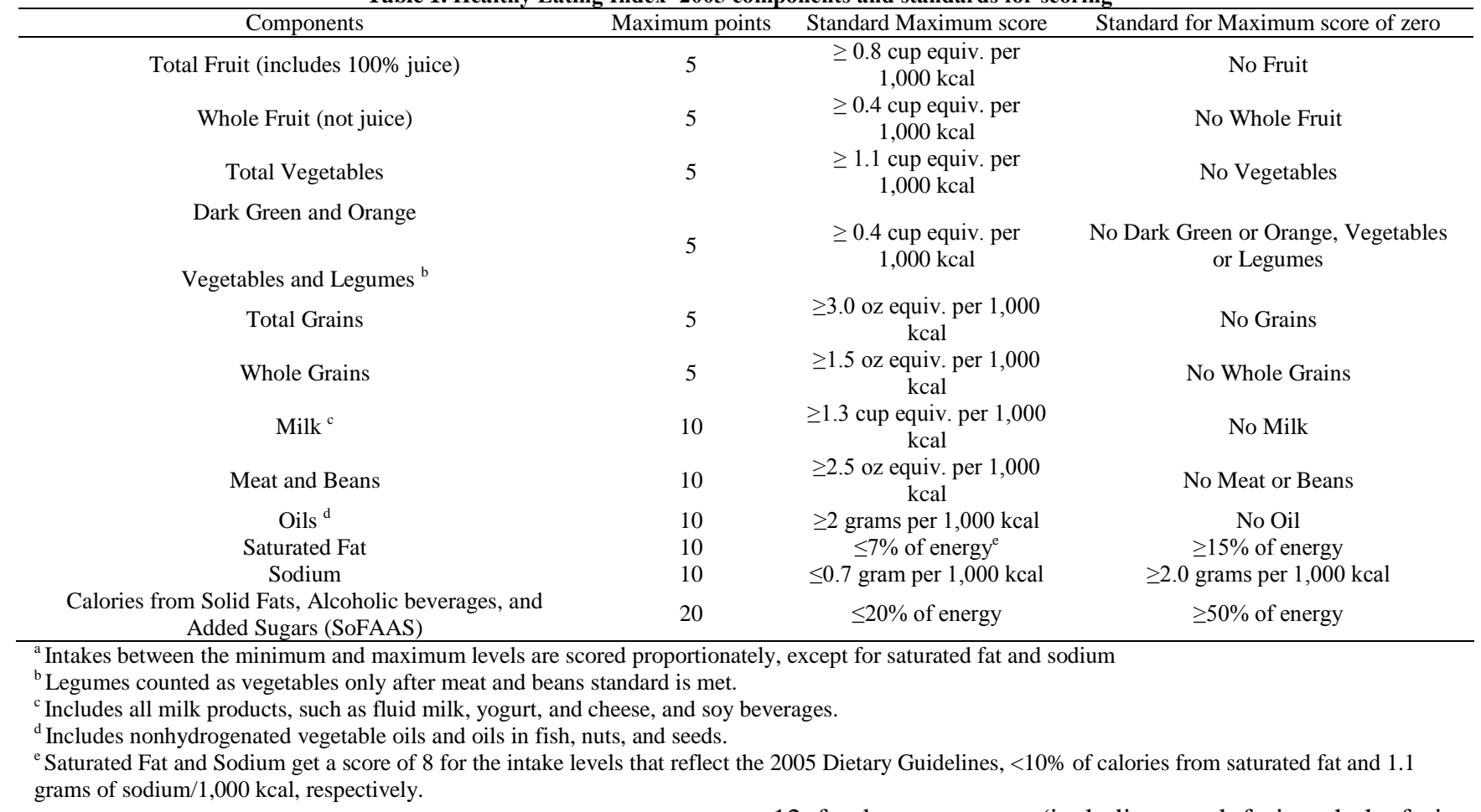

\subsection{Calculation of Healthy Eating Index-2005 Score}

In this study, the Healthy Eating Index (HEI-2005) has been used to evaluate diet quality. The HEI-2005 contains
12 food components (including total fruit, whole fruit, total vegetable, dark green and orange vegetable and legumes, total grain, whole grain, milk, meat and beans, oil, saturated fat, sodium, and percent of calorie intake from solid fats, alcoholic beverages, and added sugars (SoFAAS)). The maximum score in HEI-2005 is 100 and 
higher scores indicate a better diet quality. In this index, dietary intake recommendations are expressed per 1000 kilocalories energy intake for all components except for saturated fat and SoFAAS. Since alcohol consumption has been prohibited for adolescents in DGA-2005, percent of calorie intake from alcohol was excluded from the last component of this index and only percent of calorie intake from solid fats and added sugars was calculated as SoFAAS.

\subsection{Clinical and Biological Measurement}

Anthropometric measures were preformed according to standard protocol. Weight was measured using digital scales (seca, Hamburg Germany), with subjects minimally clothed and without shoes and was recorded to the nearest 100 g. Height was measured in standing position, without shoes and with their shoulders in a normal status, using a tape meter fixed to a wall and recorded to the nearest 0.5 cm. Waist Circumference (WC) was measured with an unstretched tape meter, at the umbilical level over light clothing, without any pressure to body surface and was recorded to the nearest $0.1 \mathrm{~cm}$.

To measure blood pressure, participants were first asked to rest for $15 \mathrm{~min}$, after which, while the subjects were in a seated position, a qualified physician measured blood pressure twice, using a standard mercury sphygmomanometer, after one initial measurement to determine peak inflation level. There was at least a 30 seconds interval between these two separate measurements, and the mean of 2 measurements was defined as the participant's blood pressure. The systolic blood pressure (SBP) was defined as the appearance of the first sound (korotkoff phase 1), and diastolic blood pressure (DBP) was defined as the disappearance of the sound (korotkoff phase 5) during deflation of the cuff at a 2- to 3-mm/s decrement rate of the mercury column [23].

After 12 to 14 hours of fasting overnight, between 7:00 and 9:00 AM, a fasting blood sample was drawn from all participants for measurement of glucose and lipid concentrations and centrifuged within 30 to 45 minutes. Fasting blood glucose (FBS) was measured on the day of blood collection by enzymatic colorimetric method using glucose oxidase. Triglyceride (TG) concentration was measured using commercially available enzymatic reagent (Pars Azemoon, Tehran, Iran) by enzymatic colorimetric tests and with glycerol phosphate oxidase. High-density lipoprotein-cholesterol (HDL-C) was measured after precipitation of the apolipoprotein B-containing lipoproteins with phosphotungstic acid. Inter- and intraassay coefficients of variation were $1.6 \%$ and $0.6 \%$, respectively, for TG and $2 \%$ and $0.5 \%$ for HDL-C $[15,24]$.

\subsection{Physical Activity Assessment}

Physical activity was assessed, using Modifiable Activity Questionnaire (MAQ) for adolescents. In this questionnaire, the frequency and duration of physical activity was questioned. To determine the physical activity level, first the intensity of each physical activity was weighted based on Metabolic Equivalent (MET). Then, for each activity, MET value, duration (in minutes) and frequency of activity per week were multiplied together and obtained numbers from various activities were added. Afterward, the physical activity level of each person was reported based on MET h/wk and subjects were classified in 3 groups; sedentary ( $3>$ MET), moderate (6>MET> 3) and vigorous $(6<\mathrm{MET})$ physical activity.

\subsection{Metabolic Syndrome and Overweight Definitions}

There is no clear consensus about the definition for MetS in adolescents. In 2010,A study conducted within the framework of TLGS showed that among different definitions of MetS for adolescents (including Cook [25], Duncan [26], Boney [27], Cruz [28] and de Ferranti [29]), the de Ferranti definition is a better predictor for MetS, overweight and obesity in early adulthood. ${ }^{6}$ Hence, in the present study, MetS was defined according to the de Ferranti criteria and was characterized as the presence of more than 3 of the following factors: Fasting blood glucose $\geq 110 \mathrm{mg} / \mathrm{dl}$, triglycerides $\geq 100 \mathrm{mg} / \mathrm{dl}$, HDL-C $<45 \mathrm{mg} / \mathrm{dl}$, for 15 to 19 year old boys and $<50 \mathrm{mg} / \mathrm{dl}$ for other boys and girls, waist circumference $>75$ th percentile for age/sex Iranian population (according to the Caspian Study [30]), and systolic or diastolic blood pressure $\geq 90$ th percentile for height, age and sex. Systolic and diastolic blood pressure percentiles were defined according to the tables published by the American Academy Of Pediatrics [31], which have been reported for 1 to 17 year olds. For subjects aged $>17$ years, the adult cut offs of the National Cholesterol Education Program (NCEP) were used [32].

In the present study, Body Mass Index (BMI) was calculated by dividing weight in kilograms to square of height in meter. At risk for overweight $(\geq 85$ th and $<95$ th percentiles) and overweight ( $\geq 95$ th percentile) were defined, based on the Iranian BMI percentiles [33].

\subsection{Statistical Analysis}

All statistical analysis were performed using the SPSS software (version 20.0) and a $\mathrm{P}_{\text {value }}<0.05$ was considered significant. All MetS risk factors were shown to have a normal distribution, except for triglycerides. To compare the characteristics and dietary intakes of participants across quartile categories of HEI-2005 score, analysis of covariance was done for continuous variables and $\chi^{2}$ test was used for dichotomous variables. In order to assess the mean values of different risk factors of MetS across HEI2005 quartiles, analysis of covariance adjusted for sex, age, energy intake and physical activity was used. Since for non-normal data the geometric mean values are reported for triglyceride, $L n$ was used to normalize the data and analysis of covariance was performed on $L n$ values. After that, by using anti-logarithms of the obtained values, geometric means were determined. The relationship between the HEI-2005 with MetS and its risk factors was calculated using logistic regression with the lowest HEI2005 quartile being considered as the reference category. To determine $\mathrm{P}$ trend within quartile categories of HEI2005, linear regression coefficient was used for continuous variables and logistic regression was used for dichotomous variables.

\section{Results}

In the present study of 706 participants aged of $14.8 \pm 2.7$ years, 54.5 percent were girls and 45.5 percent 
were boys. Generally, 22.4 percent of adolescents (20.0 percent of girls and 25.2 percent of boys) met the de Ferranti criteria for MetS. The percentages of participants with MetS and its component are shown in Table 2. Only the percentage of individuals with hypertriglyceridemia was higher in boys than girls. Large waist circumference was the most common component of MetS in both girls and boys.

Table 2. Percentage of adolescents with metabolic syndrome ${ }^{a}$ and its component: Tehran Lipid and Glucose Study

\begin{tabular}{|c|c|c|c|c|}
\hline & $\begin{array}{c}\text { total } \\
(n=706)\end{array}$ & $\begin{array}{c}\text { Girls } \\
(n=385)\end{array}$ & $\begin{array}{c}\text { Boys } \\
(n=321)\end{array}$ & Pvalue $^{\mathrm{b}}$ \\
\hline Hyperglycemia & $1.3(9)$ & $1.6(6)^{\mathrm{c}}$ & $0.9(3)$ & 0.52 \\
\hline Hypertriglyceridemia & $35.7(252)$ & $30.9(119)$ & $41.4(133)$ & 0.004 \\
\hline Low HDL-C & $47.0(332)$ & $49.4(190)$ & $44.2(142)$ & 0.20 \\
\hline Large waist circumference & $51.8(366)$ & $49.6(191)$ & $54.5(175)$ & 0.20 \\
\hline Hypertension & $11.5(81)$ & $10.4(40)$ & $12.8(41)$ & 0.34 \\
\hline Metabolic syndrome & $22.4(158)$ & $20.0(77)$ & $25.2(81)$ & 0.10 \\
\hline
\end{tabular}

${ }^{a}$ according to the de Ferranti criteria (characterized as the presence of $\geq 3$ of the following factors: fasting blood glucose $\geq 110 \mathrm{mg} / \mathrm{dl}$, triglycerides $\geq 100$ $\mathrm{mg} / \mathrm{dl}$, HDL-C $<45 \mathrm{mg} / \mathrm{dl}$ for 15 to 19 year old boys and $<50 \mathrm{mg} / \mathrm{dl}$ for others, waist circumference $>75$ th percentile for age/sex Iranian population, and systolic or diastolic blood pressure $\geq 90$ th percentile for height, age and sex)

${ }^{\mathrm{b}}$ Pvaluewas measured using chi-square

c percent (number)

Table 3. Participant characteristics according to quartile categories of the Healthy Eating Index-2005 (HEI-2005)

\begin{tabular}{|c|c|c|c|c|c|}
\hline & \multicolumn{5}{|c|}{ HEI-2005 quartile category } \\
\hline & 1 & 2 & 3 & 4 & $\mathrm{P}_{\text {trend }} \mathrm{b}$ \\
\hline HEI-2005 range $^{\text {a }}$ & $41.4-64.6$ & $64.7-70.5$ & $70.6-76.1$ & $76.2-91.0$ & \\
\hline Participants (n) & 176 & 177 & 176 & 177 & \\
\hline HEI-2005 score, median & 59.8 & 67.5 & 73.6 & 79.6 & \\
\hline Girls (\%) & 51.7 & 53.7 & 56.2 & 56.5 & 0.40 \\
\hline Age (years) & 14.4 & 14.7 & 14.7 & 15.2 & 0.01 \\
\hline At risk for overweight (\%) & 17.0 & 25.4 & 19.3 & 23.2 & $0.0 \mathrm{c}$ \\
\hline Overweight (\%) & 20.5 & 14.1 & 10.2 & 15.8 & $0.07^{c}$ \\
\hline BMI $\left(\mathrm{kg} / \mathrm{m}^{2}\right)$, mean & 22.3 & 22.0 & 21.4 & 22.0 & 0.35 \\
\hline Physical activity score $(\mathrm{MET})^{\mathrm{e}}$, mean & 5.61 & 7.51 & 6.88 & 8.46 & 0.11 \\
\hline
\end{tabular}

${ }^{\mathrm{a}}$ scores range from 0 to 100 possible points

${ }^{\mathrm{b}} \mathrm{P}$ for trend was determined using linear regression for continuous variables and logistic regression for dichotomous variables

${ }^{\mathrm{c}} \mathrm{P}$ value for at risk for overweight and overweight was determined using chi-square

${ }^{\mathrm{d}} \mathrm{BMI}$ and physical activity score was adjusted for sex and age

e Metabolic Equivalents

Table 4. Mean (SE) dietary intake of participants across the Healthy Eating Index-2005 (HEI-2005) quartile categories ${ }^{\text {a }}$

\begin{tabular}{|c|c|c|c|c|c|}
\hline & \multicolumn{5}{|c|}{ HEI-2005 quartile category } \\
\hline & 1 & 2 & 3 & 4 & $\mathrm{P}_{\text {trend }} \mathrm{b}$ \\
\hline HEI-2005 range & $41.4-64.6$ & $64.7-70.5$ & $70.6-76.1$ & $76.2-91.0$ & \\
\hline Total reported energy(kcal/d) & $2767(62)$ & $2791(62)$ & $2694(62)$ & $2709(62)$ & 0.45 \\
\hline Carbohydrate (\% of energy intake) & $53.0(0.5)$ & $55.2(0.5)$ & $57.2(0.5)$ & $57.3(0.5)$ & $<0.001$ \\
\hline Protein (\% of energy intake) & $12.7(0.2)$ & $13.5(0.2)$ & $13.7(0.2)$ & $14.7(0.2)$ & $<0.001$ \\
\hline Fat (\% of energy intake) & $34.3(0.4)$ & $31.3(0.4)$ & $29.1(0.4)$ & $28.0(0.4)$ & $<0.001$ \\
\hline Trans (\% of energy intake) & $1.85(0.06)$ & $1.34(0.06)$ & $1.17(0.06)$ & $0.88(0.06)$ & $<0.001$ \\
\hline Saturated fat ( \% of energy intake) & $12.00(0.20)$ & $10.86(0.20)$ & $9.36(0.20)$ & $9.21(0.20)$ & $<0.001$ \\
\hline Sodium (mg) & $4284(72)$ & $4065(72)$ & $3816(72)$ & 3385 (72) & $<0.001$ \\
\hline Total fiber (gr) & 16.8 & $16.9(0.44)$ & $18.1(0.44)$ & $18.0(0.44)$ & 0.003 \\
\hline Grain (oz eq/day) & $12.5(0.35)$ & $11.7(0.35)$ & $11.7(0.35)$ & $10.4(0.35)$ & 0.002 \\
\hline Whole grain (oz eq/day) & $5.50(0.33)$ & $5.50(0.33)$ & $5.82(0.33)$ & $4.85(0.33)$ & 0.97 \\
\hline Meat ${ }^{\mathrm{c}}$ (oz eq/day) & $3.35(0.14)$ & $3.92(0.13)$ & $4.38(0.14)$ & $5.47(0.14)$ & $<0.001$ \\
\hline Fruit (cup eq/day) & $1.57(0.17)$ & $2.61(0.17)$ & $3.44(0.17)$ & $3.71(0.17)$ & $<0.001$ \\
\hline Vegetables ${ }^{\mathrm{d}}$ (cup eq/day) & $2.68(0.10)$ & $2.93(0.10)$ & $3.09(0.10)$ & $3.59(0.10)$ & $<0.001$ \\
\hline Dairy products (cup eq/day) & $2.38(0.10)$ & $2.56(0.10)$ & $2.16(0.10)$ & $2.46(0.10)$ & 0.69 \\
\hline Calories from solid fats and added sugars (\% of energy intake) & $27.2(0.4)$ & $23.1(0.4)$ & $20.0(0.4)$ & $18.0(0.4)$ & $<0.001$ \\
\hline
\end{tabular}

${ }^{\mathrm{a}}$ Values are determined using ANCOVA after adjustment for age, sex and energy intake

${ }^{\mathrm{b}} \mathrm{P}$ for trend was determined using linear regression

${ }^{\mathrm{c}}$ According to the dietary guidelines for Americans recommendations, legumes were assigned to the meat group for those who have not achieved maximum points of meats, and if the meat point was maximized an extra amount was counted in the vegetable group

${ }^{\mathrm{d}}$ Including vegetables and legumes

e Including fat from plant and fish source

The mean score of HEI-2005 in the present study was

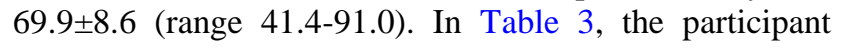
characteristics according to quartiles of HEI were shown. Based on HEI-2005, the diet quality score was higher in older subjects, but there was no significant difference in sex, BMI and physical activity scores between the highest and lowest quartile categories.

Table 4 shows the dietary intake of participants across the HEI-2005 quartile categories. In the highest HEI-2005 quartile category, after adjustment for sex, age and energy intake, the percent of energy intake from total fat, trans fatty acids, saturated fatty acid, solid fat and added sugar and also sodium and grain intake were significantly lower than those in the lowest quartile category ( $\mathrm{p}<0.001$ ).

Adjusted mean values of MetS components, in their continuous form, across the HEI-2005 quartiles are given in Table 5. After adjustment for sex, age, energy intake and physical activity, fasting blood glucose and 
triglyceride showed a significant decreasing trend and HDL-C showed a significant increasing trend according to quartiles of HEI-2005. However, after further adjustment for BMI, decreasing trends of fasting blood glucose and triglyceride concentrations across HEI-2005 quartiles were not significant (data were not shown). HEI-2005 score did not show a significant association with large waist circumference or hypertension.

When MetS and its components were considered as dichotomous variables, those in the highest HEI-2005 category had significantly lower odds of hypertriglyceridemia (OR: 0.62; 95\% CI: 0.40-0.96; P trend=0.03) and low HDL-C (OR: 0.63; 95\% CI: 0.410.97; P trend=0.03) than did those in the lowest HEI-2005 category after adjustment for sex, age, energy intake and physical activity (Table 6). Further adjustment for BMI did not have any effect on this association (data not shown). No significant difference was observed in odds of MetS, large waist circumference, hyperglycemia and hypertension.

Table 5. Mean (SE) for components of metabolic syndrome in participants across the Healthy Eating Index-2005 (HEI-2005) quartile categories $^{\mathrm{a}}$

\begin{tabular}{|c|c|c|c|c|c|}
\hline & \multicolumn{5}{|c|}{ HEI-2005 quartile category } \\
\hline & 1 & 2 & 3 & 4 & $\mathrm{P}_{\text {trend }} \mathrm{b}$ \\
\hline HEI-2005 range & $41.4-64.6$ & $64.7-70.5$ & $70.6-76.1$ & $76.2-91.0$ & \\
\hline Fasting blood glucose (mg/dl) & $93.1(0.6)$ & $92.7(0.6)$ & $92.0(0.6)$ & $91.7(0.6)$ & 0.04 \\
\hline Triglycerides $(\mathrm{mg} / \mathrm{dl})^{\mathrm{c}}$ & $93.0(1.2)$ & $89.4(1.1)$ & $82.5(1.2)$ & $84.1(1.2)$ & 0.03 \\
\hline HDL-C (mg/dl) & $48.6(0.8)$ & $49.5(0.8)$ & $50.3(0.8)$ & $50.9(0.8)$ & 0.01 \\
\hline Waist circumference (cm) & $77.8(0.9)$ & $77.7(0.9)$ & $75.5(0.9)$ & $76.5(0.9)$ & 0.23 \\
\hline Systolic blood pressure (mmHg) & $102.1(0.9)$ & $102.1(0.9)$ & $101.0(0.9)$ & $101.2(0.9)$ & 0.72 \\
\hline Diastolic blood pressure (mmHg) & $67.3(0.7)$ & $67.7(0.7)$ & $66.4(0.7)$ & $67.3(0.7)$ & 0.58 \\
\hline
\end{tabular}

\footnotetext{
${ }^{\mathrm{a}}$ Values are determined using ANCOVA after adjustment for age, sex, energy intakes and physical activity

${ }^{\mathrm{b}} \mathrm{P}$ for trend was determined using linear regression

${ }^{\mathrm{c}}$ for triglyceride, geometric mean values are reported.
}

Table 6.Odds ratio (95\% CI) for metabolic syndrome a and its components across the Healthy Eating Index-2005 (HEI-2005) quartile categories in Tehran Lipid and Glucose Study adolescents ${ }^{\text {b }}$

\begin{tabular}{|c|c|c|c|c|c|}
\hline & \multicolumn{5}{|c|}{ HEI-2005 quartile category } \\
\hline & 1 & 2 & 3 & 4 & $\mathrm{P}_{\text {trend }}{ }^{\mathrm{c}}$ \\
\hline HEI-2005 range & $41.4-64.6$ & $64.7-70.5$ & $70.6-76.1$ & $76.2-91.0$ & \\
\hline Metabolic syndrome $^{\mathrm{c}}$ & 1 & $0.73(0.45-1.19)$ & $0.72(0.44-1.18)$ & $0.69(0.40-1.11)$ & 0.11 \\
\hline Hyperglycemia & 1 & $0.70(0.11-4.24)$ & $1.00(0.20-5.12)$ & $0.38(0.04-0.80)$ & 0.54 \\
\hline Hypertriglyceridemia & 1 & $0.68(0.44-1.05)$ & $0.62(0.40-0.97)$ & $0.62(0.40-0.96)$ & 0.03 \\
\hline Low serum HDL-C & 1 & $0.82(0.54-1.23)$ & $0.70(0.46-1.07)$ & $0.63(0.41-0.97)$ & 0.03 \\
\hline Large waist circumference & 1 & $0.91(0.60-1.39)$ & $0.72(0.47-1.10)$ & $0.80(0.52-1.23)$ & 0.18 \\
\hline Hypertension & 1 & $1.06(0.58-1.95)$ & $0.70(0.36-1.36)$ & $0.61(0.30-1.24)$ & 0.11 \\
\hline
\end{tabular}

a according to the de Ferranti criteria (characterized as the presence of $\geq 3$ of the following factors: fasting blood glucose $\geq 110 \mathrm{mg} / \mathrm{dl}$, triglycerides $\geq 100$ $\mathrm{mg} / \mathrm{dl}$, HDL-C $<45 \mathrm{mg} / \mathrm{dl}$ for 15 to 19 years old boys and $<50 \mathrm{mg} / \mathrm{dl}$ for others, waist circumference $>75$ th percentile for age/sex Iranian population, and systolic or diastolic blood pressure $\geq 90$ th percentile for height, age and sex)

${ }^{\mathrm{b}}$ Values are adjusted for age, sex, energy intake and physical activity

${ }^{\mathrm{c}} \mathrm{P}$ for trend was determined using logistic regression

\section{Discussion}

In this population-based study, we evaluated the relationship between diet quality (based on Healthy Eating Index-2005) and MetS in adolescents. Our findings showed that participants whose dietary patterns were in close concordance with HEI-2005 had lower odds of hypertriglyceridemia and low HDL-C, independent of sex, age, energy intake and physical activity. Also we found no significant relationship between HEI-2005 score and MetS.

Some studies have investigated the relationship between dietary intake and MetS risk factors in adolescents. Pan and Pratt [14] have shown inverse relationship between overall HEI score and HEI fruit score with MetS $(\mathrm{P}<0.001)$; however, after adjustment for age, sex, ethnicity, poverty status, BMI and physical activity, only the HEI fruit score showed a significant inverse relationship with MetS. According to the Linardakis and colleagues [1], numbers of existing MetS components and systolic blood pressure were decreased with increase in diet quality of adolescents based on HEI, whereas no significant difference was observed in diastolic blood pressure, waist circumference or blood concentrations of glucose, triglycerides and HDL-C. In addition, it has been shown that high adherence to the Mediterranean Diet (MD) was associated with low prevalence of MetS, hypertriglyceridemia and low HDL-C in adolescents [34]. However, in Iranian adolescents, increasing adherence to Dietary Guidelines for Americans Adherence Index (DGAI) had an inverse association with low HDL-C and hypertension, but had no relationship with the risk of MetS and its other components [35].

Studies conducted in Iranian and American adults $[36,37]$ have shown that with increasing adherence to DGAI, 21\% and 36\% reduction were observed in MetS, respectively. These reductions were also observed in some of MetS components. DGAI, similar to HEI-2005, was developed as an index of adherence to the DGA-2005 [12]. However, in Iranian adults, no significant association was observed between HEI-2005 and serum concentration of triglycerides and HDL-C, as well as waist circumference [38,39].

In the present study, there was no significant association between HEI-2005 scores and odds of MetS. Also, no significant difference was observed in odds of high waist circumference, the percentage of people at risk for overweight or overweight, and the mean physical activity scores across HEI-2005 quartile categories. Despite the fact that energy intake is one of the factors affecting overweight and high waist circumference, our findings suggest that HEI-2005 is unable to control the effect of excess food and energy consumption. The energy density approach of this index focuses on intakes of food 
groups and nutrients per 1000 kilocalories energy intake and does not consider the individual energy and food groups needs. Although this approach results in balance among food group intakes, it does not consider controlled energy consumption [39]. In addition, previous studies have shown that the prevalence of MetS is higher in overweight adolescents, compared to those with normal BMI [8,34,40]. Therefore, lack of any significant association between HEI-2005 and MetS might be potentially related to the inability of this index to control the effect of over-consumption, and hence overweight and large waist circumference.

The results of our present research do not show an association between hyperglycemia and the HEI-2005 score, similar to the results of other researches on children and adolescents, which showed no relationship between HEI and MD with hyperglycemia [1,34]. Due to the low prevalence of hyperglycemia in children and adolescents, it would be controversial to consider this as one of MetS components in this age group [1]; hence, the lack of any association in our study could be due to the low percentage of subjects with hyperglycemia (1.3\%).

Previous studies have indicated an inverse relationship for HDL-C levels with fruit and vegetable and fiber intake $[41,42,43]$. Isfahan Healthy Heart Study [44] reported that high intake of saturated fat is associated with dyslipidemia in Iranian youth. Therefore, higher intake of fiber, fruit, and vegetables along with lower intake of saturated fatty acid in the higher quartile categories of HEI-2005 could be plausible biological mediators for our observed association of these metabolic risk factors.

It is worth mentioning that the findings of this research are subject to several potential limitations. Since there are no dietary guidelines available for Iranian populations, adolescent's dietary patterns were assessed by the HEI2005 which is based on dietary guidelines for Americans. In fact, our investigation aimed to introduce an index to assess the dietary pattern of Iranian adolescents. Our findings showed that despite the associations observed between HEI-2005 and some metabolic disorders, this index could not control the effect of over-consumption. Therefore, some modifications are required to customize this index to be used in our population. It is thought that taking maximum cut-offs for some items and giving negative scores to those with higher intake than the cutoffs could be useful to better adjust the index for our population. However, HEI-2005 does not contain recommendations on body weight and physical activity components of DGA which could affect the relationship between dietary pattern and chronic disease. In addition, cross-sectional studies can not accurately indicate the extent of adherence to dietary recommendations or the effect of diet quality on the development of MetS.

One of the strengths of our study is that it is a population-based study conducted in a developing country under nutrition transition. To the best of our knowledge, this is the first study that evaluates the association of diet quality and MetS prevalence in Iranian adolescents. Moreover, measurement and control of many known confounders can be considered as other strengths of the present study.

In conclusion, elucidation of how dietary pattern is associated with MetS risk factors is valuable. Taken collectively, our findings indicate that adherence to the
HEI-2005 only reduce the odds of hypertriglyceridemia and low HDL-C. Future studies, including dietary intervention studies, will be needed to confirm the effects of the HEI-2005 dietary patterns on intermediate markers of disease and on the prevention of new cases of disease.

\section{Acknowledgment}

We are grateful to the TLGS participants and personnel for their collaboration. This study was supported by a grant from the Research Institute of Endocrine Sciences, Shahid Beheshti University Medical Sciences, Tehran, Iran. The authors wish to thank MS. Niloofar Shiva for critical editing of English grammar and syntax of the manuscript.

\section{References}

[1] Linardakis M, Bertsias G, Sarri K, Papadaki A, Kafatos A, "Metabolic syndrome in children and adolescents in Crete, Greece, and association with diet quality and physical fitness", J Public Health, 16 (6), 421-428, 2008.

[2] Esposito K, Giugliano D, "Diet and inflammation: a link to metabolic and cardiovascular diseases”, Eur Heart, 27 (1), 15-20, 2006.

[3] Kelishadi R, "Childhood overweight, obesity, and the metabolic syndrome in developing countries” Epidemiol Rev, 29 (1), 62-76, 2007.

[4] Fakhrzadeh H, Ebrahimpour P, Pourebrahim R, Heshmat R, Larijani B, "Metabolic Syndrome and its Associated Risk Factors in Healthy Adults: APopulation-Based Study in Iran", Metabolic syndrome and related disorders, 4 (1), 28-34 2006.

[5] Kelishadi R, Ardalan G, Gheiratmand R, Adeli K, Delavari A, Majdzadeh R, "Paediatric metabolic syndrome and associated anthropometric indices: the CASPIAN Study", Acta Paediatrica, 95 (12), 1625-1634, 2006.

[6] Mirmiran P, Sherafat-Kazemzadeh R, Farahani SJ, Asghari G, Niroomand M, Momenan AA, et al, "Performance of different definitions of metabolic syndrome for children and adolescents in a 6-year follow-up: Tehran Lipid and Glucose Study (TLGS)”, Diabetes Res ClinPract, 89 (3), 327-333, 2010.

[7] Chiti H, Hoseinpanah F, Mehrabi Y, Azizi F, "The Prevalence of Metabolic Syndrome in Adolescents with Varying Degrees of Body Weight: Tehran Lipid and Glucose Study (TLGS)”, IJEM, 11 (6), 625-637, 2009 [in persian].

[8] De Ferranti SD, Osganian SK, "Epidemiology of paediatric metabolic syndrome and type 2 diabetes mellitus” Diabetes and Vascular Disease Research, 4 (4), 285-296, 2007.

[9] Mirmiran P, Azadbakht L, Azizi F, "Healthy Eating Index and its Relation with Diet Quality in Tehrani Adolescents: Tehran Lipid and Glucose Study”, RJMS, 11 (43), 829-838, 2004 [in persian].

[10] Hu, F. B, "Dietary pattern analysis: a new direction in nutritional epidemiology”, Current opinion in lipidology, 13 (1), 3-9, 2002.

[11] Grundy SM, Hansen B, Smith Jr SC, Cleeman JI, Kahn RA, "Clinical management of metabolic syndrome”, Circulation, 109 (4), 551-556, 2004.

[12] Fogli-Cawley JJ, Dwyer JT, Saltzman E, McCullough ML, Troy LM, Jacques PF, "The 2005 dietary guidelines for Americans adherence index: development and application”, J Nutr, 136 (11), 2908-2915, 2006.

[13] Guenther PM, Reedy J, Krebs-Smith SM, "Development of the healthy eating index-2005”, J Am Diet Assoc, 108 (11), 189618901, 2008.

[14] Pan Y, Pratt CA, "Metabolic syndrome and its association with diet and physical activity in US adolescents”, J Am Diet Assoc, 108 (2), 276-286, 2008.

[15] Azizi F, Ghanbarian A, Momenan AA, Hadaegh F, Mirmiran P, Hedayati $\mathrm{M}$, et al, "Prevention of non-communicable disease in a population in nutrition transition: Tehran Lipid and Glucose Study phase II”, Trials, 10 (5), 1-15, 2009.

[16] Azizi F, Rahmani M, Emami H, Mirmiran P, Hajipour R, Madjid $\mathrm{M}$, et al, "Cardiovascular risk factors in an Iranian urban 
population: Tehran lipid and glucose study (phase 1)" Sozial-und Präventivmedizin/Social and Preventive Medicine, 47 (6), 408-426, 2002.

[17] Willett W, Nutritional epidemiology, Issues in Analysis and Presentation of Dietary Data, 1998, 321-347.

[18] Hosseini Esfahani F, Asghari G, Mirmiran P, Azizi F, "Reproducibility and relative validity of food group intake in a food frequency questionnaire developed for the Tehran lipid and glucose study”, J Epidemiol, 20 (2), 150-158, 2010.

[19] Mirmiran P, Hosseini Esfahani F, Mehrabi Y, Hedayati M, Azizi F, "Reliability and relative validity of an FFQ for nutrients in the Tehran Lipid and Glucose Study” Public Health Nutr, 13 (5), 654662, 2010.

[20] Asghari G, Rezazadeh A, Hosseini-Esfahani F, Mehrabi Y, Mirmiran P, Azizi F, "Reliability, comparative validity and stability of dietary patterns derived from an FFQ in the Tehran Lipid and Glucose Study” British Journal of Nutrition, 108 (06), 1109-1117, 2012.

[21] Azar M, Sarkisian E, Food Composition Table of Iran, National Nutrition and Food Research Institute. Shahid Beheshti University Press, Tehran, 1980.

[22] The Nutrient Data Laboratory. Food Composition Table (FCT) food and nutrition information center, United States Department of Agriculture (USDA). Available from: URL: http://www.nal.usda.gov/fnic/foodcomp

[23] Ghanbarian A, Rezaei-Ghaleh N, Salehi P, Azizi F, "Blood Pressure Distribution in an Iranian Adolescent Population: IITehran Lipid and Glucose Study” Med J Malaysia, 61 (4), 433437, 2006.

[24] Azizi F, Rahmani M, Madjid M, Allahverdian S, Ghanbili J, Ghanbarian A, et al, "Serum lipid levels in an Iranian population of children and adolescents: Tehran lipid and glucose study”, Eur J Epidemiol, 17 (3), 281-288, 2001.

[25] Cook S, Weitzman M, Auinger P, Nguyen M, Dietz WH, "Prevalence of a metabolic syndrome phenotype in adolescents: findings from the third National Health and Nutrition Examination Survey, 1988-1994” Arch PediatrAdolesc Med, 157 (8), 821-827, 2003.

[26] Duncan GE, Li SM, Zhou X-H, "Prevalence and trends of a metabolic syndrome phenotype among US adolescents, 19992000”, Diabetes Care, 27 (10), 2438-2443, 2004.

[27] Boney CM, Verma A, Tucker R, Vohr BR, "Metabolic syndrome in childhood: association with birth weight, maternal obesity, and gestational diabetes mellitus”, Pediatrics, 115 (3), 290-296, 2005.

[28] Cruz ML, Goran MI, "The metabolic syndrome in children and adolescents”, Current Diabetes Reports, 4, 53-62, 2004

[29] de Ferranti SD, Gauvreau K, Ludwig DS, Neufeld EJ, Newburger JW, Rifai N, "Prevalence of the metabolic syndrome in American adolescents” Circulation, 110 (16), 2494-2497, 2004

[30] Kelishadi R, Gouya MM, Ardalan G, Hosseini M, Motaghian M, Delavari A, et al, "First reference curves of waist and hip circumferences in an Asian population of youths: CASPIAN study” J Trop Pediatr, 53 (3), 158-164, 2007.
[31] American Academy of Pediaterics, "The fourth report on the diagnosis, evaluation, and treatment of high blood pressure in children and adolescents. Pediatrics”, 114 (2), 555-576, 2004.

[32] Grundy SM, Cleeman JI, Daniels SR, Donato KA, Eckel RH, Franklin BA, et al, "Diagnosis and management of the metabolic syndrome: an American Heart Association/National Heart, Lung, and Blood Institute Scientific Statement”, Circulation, 112 (17), 2735-52, 2005.

[33] Kelishadi R, Ardalan G, Gheiratmand R, Majdzadeh R, Hosseini M, Gouya M, et al, "Thinness, overweight and obesity in a national sample of Iranian children and adolescents: CASPIAN Study” Child Care Health Dev, 34 (1), 44-54, 2008.

[34] Bibiloni MM, Martínez E, Llull R, Maffiotte E, Riesco M, Llompart I, et al, "Metabolic syndrome in adolescents in the Balearic Islands, a Mediterranean region”, Nutr Metab Cardiovasc Dis, 21 (6), 446-454, 2009.

[35] Mohseni-Takalloo S, Mirmiran P, Mehrabi Y, Hosseini-Esfahani F, Azizi F, "Diet Quality and its Relationship with Metabolic Syndrome in Adolescents: Tehran Lipid and Glucose Study", Iranian Journal of Endocrinology and Metabolism, 15 (1), 3-13, 2013.

[36] Hosseini-Esfahani F, Jessri M, Mirmiran P, Bastan S, Azizi F, "Adherence to dietary recommendations and risk of metabolic syndrome: Tehran Lipid and Glucose Study”, Metabolism, 59 (12) 1833-1842, 2010.

[37] Fogli-Cawley JJ, Dwyer JT, Saltzman E, McCullough ML, Troy LM, Meigs JB, et al, “The 2005 Dietary Guidelines for Americans and risk of the metabolic syndrome”, Am J Clin Nutr, 86 (4), 1193-1201, 2007.

[38] Asghari G, Mirmiran P, Hosseni-Esfahani F, Nazeri P, Mehran M, Azizi F, "Dietary quality among Tehranian adults in relation to lipid profile: Findings of the Tehran Lipid and Glucose Study”, $J$ HEALTH POPUL NUTR, 31 (1), 37-48, 2013.

[39] Asghari G, Mirmiran P, Rashidkhani B, Asghari-Jafarabadi M, Mehran M, Azizi F, "The association between diet quality indices and obesity: Tehran Lipid and Glucose Study”, Arch Iran Med, 15 (10), 599-605, 2012.

[40] Li Y, Yang X, Zhai F, Kok F, Zhao W, Piao J, et al, "Prevalence of the metabolic syndrome in Chinese adolescents", Br J Nutr, 99 (3), 565-570, 2008.

[41] Chen WJL, Anderson JW, "Effects of plant fiber in decreasing plasma total cholesterol and increasing high-density lipoprotein cholesterol”, ExpBiol Med, 162 (2), 310-313, 1979.

[42] Toeller M, Buyken AE, Heitkamp G, De Pergola G, Giorgino F, Fuller JH, "Fiber intake, serum cholesterol levels, and cardiovascular disease in European individuals with type 1 diabetes”, Diabetes care, 22 (2), 21-28, 1999.

[43] Bertsias G, Linardakis M, Mammas I, Kafatos A, "Fruit and vegetables consumption in relation to health and diet of medical students in Crete, Greece”, Journal international de vitaminologie et de nutrition, 75 (2),107-117, 2005.

[44] Kelishadi R, Pour MH, Zadegan NS, Kahbazi M, Sadry G, Amani A, et al, "Dietary fat intake and lipid profiles of Iranian adolescents: Isfahan Healthy Heart Program--Heart Health Promotion from Childhood”, Prev Med, 39 (4), 760-766, 2004. 